\title{
Bioinformation
}

\author{
www.bioinformation.net
}

Views \& Challenges

\section{RNAi and RNAa - The Yin and Yang of RNAome}

\author{
Peter Natesan Pushparaj ${ }^{1, *}$, Jude J Aarthi ${ }^{1}$, Srinivasan D Kumar ${ }^{2}$, Jayapal Manikandan ${ }^{1}$ \\ ${ }^{1}$ Department of Physiology, Yong Loo Lin School of Medicine, National University of Singapore, Singapore-119260; ${ }^{2}$ Department of \\ Anatomy, Yong Loo Lin School of Medicine, National University of Singapore, Singapore-119260; \\ Peter Natesan Pushparaj* - E-mail: phspnp@nus.edu.sg; Phone: +65-90103795; * Corresponding author
}

received December 22, 2007; revised December 26, 2007; accepted January 02, 2008; published online January 11, 2008

\begin{abstract}
:
RNA interference (RNAi) is a powerful technology with huge applications for functional genomics, target identification in drug discovery and elucidation of molecular signaling pathways. Current RNAi studies have demonstrated the clinical potential of small interfering RNAs (siRNAs) in metabolic diseases, cancer, AIDS, malaria, neurodegenerative disorders, dental diseases and other illnesses. Interestingly, recent studies have shown that the small RNA molecules, either indigenously produced as microRNAs (miRNAs) or exogenously administered synthetic dsRNAs could effectively activate a particular gene in a sequence specific manner instead of silencing it. This novel, but still uncharacterized, phenomenon has been termed as RNA activation (RNAa). The paradoxical concept of Yin and Yang, which describe two primal opposing but complementary principles, can potentially be applied to elucidate the complex phenomenon of RNAa/RNAi in the RNAome. This warrants a proper understanding of the RNAi/RNAa molecular pathways in living organisms before any of the small dsRNAs can potentially be exploited for therapeutics in human beings.
\end{abstract}

Key Words: RNA interference; RNA activation; Yin and Yang; RNAome; small interfering RNA; gene silencing; functional genomics; drug discovery; therapeutics

\section{Background:}

RNA interference (RNAi) is a precise and powerful gene knock down mechanism conserved in evolution from Caenorhabditis elegans to higher mammals. It was first experimentally shown in C.elegans and identified that 21to 25-nucleotide short interfering RNAs (siRNAs) are the key factors of RNAi [1-2]. This pioneering experimental finding of RNAi phenomenon in C.elegans by Fire et al was duly recognized and they were awarded with Nobel Prize for Physiology or Medicine in 2006 [3]. This gene knock down phenomenon was subsequently observed and experimentally proved in flies [4-6], and vertebrates [7-8]. siRNAs theoretically represent ideal drugs for the specific inhibition of unwanted or pathogenic gene products. The market for RNAi-based drugs as a whole could be enormous. There is no disease that affects humans that doesn't have a genetic component, and RNAi sequences can be tailored to block just about any gene [9]. They make it possible by binding and then degrading the mRNA produced by the gene before that mRNA can start producing a harmful protein, which actually causes the illness. And this makes the RNAi a powerful tool with enormous applications for functional genomics, druggable molecular target identification and elucidation of molecular signaling pathways, which might possibly leads to drug discovery, and the development of therapies for various human diseases/disorders in the near future $[5,10,11]$. So far, more than 11000 articles and 1200 reviews were already published on RNAi and related fields (as cited in the pubmed in December 2007).

\section{Yin and Yang of RNAome}

An improved understanding of RNAi has provided novel tools for conducting functional studies in a gene-specific approach. So far, RNAi has been used to generate model systems, to identify novel molecular targets, to study gene function in a genome-wide manner, and to create a new niche for clinical therapeutics [12-14]. We and others have recently reviewed the therapeutic potential of synthetic siRNAs in various human diseases and disorders [10, 1214]. We have enlisted the potential siRNA targets for treating viral, neurodegenerative, cancer and other diseases [10]. The RNAi mechanism [10] can be exploited to knock down crucial disease gene or multiple disease genes, downstream signaling molecules capable of modulating a particular disease, and dominant genes which can lead to a genetic disease/ disorder [10].

Some proof of concept experiments have precisely demonstrated that the siRNA efficiency/efficacy largely depends on multiple factors (not based on any single factor), such as the thermodynamic stability of the duplex at the 5' AS end, ability to form internal hair-pins in the RISC 


\section{www.bioinformation.net}

\section{Views \& Challenges}

complex (reduces the silencing ability), GC content, base preference for an Adenosine not cytosine at $19^{\text {th }}$ or $20^{\text {th }}$ base position of the sense strand (required for unwinding/activation step in the RISC), uridine is preferred at position 10, adenosine is preferred at position 3 and any base at positon 13 except guanosine. These factors are thought to play a role in the gene silencing ability of synthetic siRNAs and make the designing of siRNAs a complex and strenuous process [15-17]. Recently, Hong et al (2005) tested the expression inhibition of transgene of HBV surface antigen by different amounts of siRNA in the presence or absence of mouse orthologue of enhanced RNA interference-1 (eri-1). Dose-response analyses carried out in vitro and in vivo using Escherichia-coli-expressed and enzyme-digested siRNA (esiRNA) confirmed the dose dependent effect. However, the results revealed an unexpected decrease of inhibition effect that was associated with the introduction of higher dose of esiHBVP, compared with lower dose of esiHBVP. They concluded that RNAi machinery may be under negative regulation through the induction of a series of genes coding for destabilizing enzymes by siRNAs introduced into the cell [18].

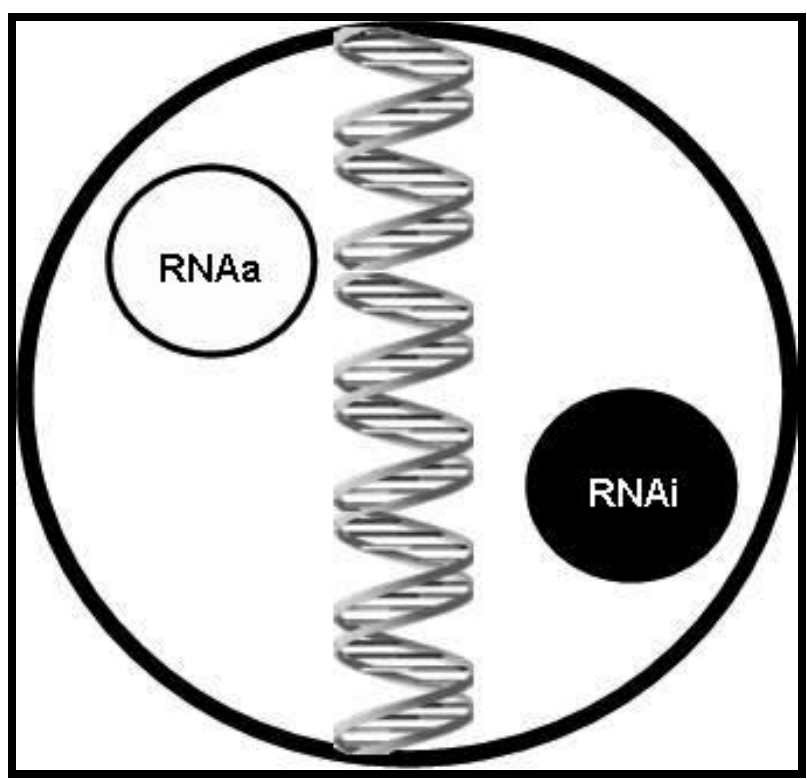

Figure 1: RNAa/RNAi constitutes the Yin and Yang of the RNAome. The dual concepts of yin and yang which describe two primal opposing but complementary principles or cosmic forces said to be found in all non-static objects and processes in the universe. This paradoxical concept can potentially be applied to elucidate the complex phenomenon of RNAa/RNAi molecular pathways in the RNAome.

By targeting the promoter region of various genes, Li et al (2006) have identified several dsRNAs that induce gene transcription in a sequence-specific manner. This RNA mediated process requires the Argonaute 2 (Ago2) protein and is associated with histone changes linked to gene activation. Although the exact mechanism is unknown at present, the identification of RNA activation (RNAa) may still have significant therapeutic application [19]. RNAa could potentially be complementing the RNAi according to the Yin and Yang Philosophy (Figure 1). The use of RNAi is currently being proposed as a gene-specific approach for molecular medicine [10-11]. By the same principle, the specific activation of silenced tumor suppressor genes such as p21 or other common dysregulated genes, such as Ecadherin, by RNAa may further add to the growing therapeutic prospective of dsRNAs-based drugs in the treatment of cancer and other diseases. Interestingly, RNAi typically silences genes for 5 to 7 days, but RNAa boosted gene activity for up to 13 days [20]. It is not well characterized that how small dsRNAs could turn genes ON, especially for a very long period. This information also indicates a new obstacle for RNAi in which siRNAs may undesirably stimulate the expression of off target genes.

\section{Conclusion:}

The molecular machinery of RNAome, involves both RNAi/RNAa, still needs to be characterized to further explain the question of how the same enzymes of RNAi pathway can sometimes turn genes OFF, and sometimes ON. Hence, a thorough understanding of the molecular machinery of RNAa and RNAi should be required before exploiting the RNA interference/activation strategies in human beings for therapeutic purposes.

\section{References:}

[01] A. Fire et al., Nature, 391: 806 (1998)

[02] P. Bastin, et al., Res Microbiol., 152: 123 (2001)

[03] J. Couzin, Science, 314: 34 (2006) 


\section{Bioinformation}

\section{www.bioinformation.net}

\section{Views \& Challenges}

\begin{tabular}{|c|c|}
\hline [04] & E. Bernstein, et al., Nature, 409: 363 (2001) \\
\hline [05] & E. Bernstein, et al., Rna, 7: 1509 (2001) \\
\hline [06] & $\begin{array}{l}\text { A. Bernards and I. K. Hariharan, Curr Opin Genet } \\
\text { Dev., 11: } 274 \text { (2001) }\end{array}$ \\
\hline [07] & G. J. Hannon, Nature, 418: 244 (2002) \\
\hline [08] & $\begin{array}{l}\text { G. J. Hannon and D. S. Conklin, Methods Mol Biol., } \\
\text { 257: } 255 \text { (2004) }\end{array}$ \\
\hline [09] & $\begin{array}{l}\text { L. Aagaard and J. J. Rossi, Adv Drug Deliv Rev., 59: } \\
75 \text { (2007) }\end{array}$ \\
\hline [10] & $\begin{array}{l}\text { P. N. Pushparaj and A. J. Melendez, Clin Exp } \\
\text { Pharmacol Physiol., 33: } 504 \text { (2006) }\end{array}$ \\
\hline [11] & J. Manikandan et al., Front Biosci., 12: 1344 (2007) \\
\hline
\end{tabular}

$\begin{array}{ll}\text { [12] } & \text { P. Y. Lu, et al., Adv Genet., 54: } 117 \text { (2005) } \\ \text { [13] } & \text { S. E. Martin and N. J. Caplen, Annu Rev Genomics } \\ & \text { Hum Genet., 8: 81 (2007) } \\ \text { [14] } & \text { F. Y. Xie, et al., Drug Discov Today, 11: } 67 \text { (2006) } \\ \text { [15] } & \text { A. Khvorova, et al., Cell, 115: 209 (2003) } \\ \text { [16] } & \text { A. Reynolds, et al., Nat Biotechnol., 22: } 326 \text { (2004) } \\ \text { [17] } & \text { Q. Boese, et al., Methods Enzymol., 392: 73 (2005) } \\ \text { [18] } & \text { J. Hong, et al., Biochem J., 390: 675 (2005) } \\ \text { [19] } & \text { L. C. Li , et al., Proc Natl Acad Sci., 103: 17337 } \\ \text { (2006) } & \text { K. Garber, Science, 314: } 741\end{array}$

Edited by P. Kangueane

Citation: Pushparaj et al., Bioinformation 2(6): 235-237 (2008)

License statement: This is an open-access article, which permits unrestricted use, distribution, and reproduction in any medium, for non-commercial purposes, provided the original author and source are credited. 